\title{
L'électrification de la ligne de Modane
}

\author{
par Marcel JAPIOT, Ingénieur en chef adjoint du Matértel de la Tracton des Chemins de fer P.-L-M.
}

\begin{abstract}
Avant de vous entretenir de ces travaux, il me paraît indispensable d'aller au devant d'une question que vous ne manqueriez certainement pas de me poser, st je ne prenais l'untiative de la soulever moi-même.

Pourquoi, me direz-vous, la Compagnie P-L -M s'est-elle bornée jusqu'ici à entreprendre l'électrtfication de la ligne de Modane, alors que les Compagntes du Mudi et d'Orléans ont déjà électrıfié de vastes portions de leurs réseaux?

Ce qui peut vous sembler une anomalte s'expltque atsément par diverses consudérations, les unes d'ordre technique, les autres d'ordre économique
\end{abstract}

Au point de vuo technique, l'électrificatıon des Chemins de fer du Midi étail lıée à l'aménagement des ressources hydraulıques des Pyrénées, et à l'installation d'un vaste réseau à haute tension pour l'interconnexion des usines et le transport de l'énergie à grande distance De même, l'adoption de la traction électrique sur les lignes de la Compagnie d'Orléans était subordonnée à la construction de puissantes usines hydroélectriques dans le Massif central, et à leur lıaison avec la région parisienne par des artères à très haute lension. Dans un cas comme dans l'autre, l'emplor de l'énergı́ électrique pour la tractıon ne constıtuait donc que l'un des aspects d'un problème beaucoup plus vaste, visant l'utilisation rationnelle d'importantes ressources hydrauliques encore inexplortées

Aucun problème de ce genre ne se posait pour la Compagnie P.-L.-M. : l'aménagement des chutes d'eau des Alpes avart été entrepris depuis fort longtemps, et se développait rapidement tundis que de nombreuses artères à haute tension transportaient déjà l'énergie à grande distance, jusqu'aux princıpaux centres de consommation. Au lıeu d'avorr à créer usınes et lignes de transport, comme le Midi dans les Pyrénées, ou l'Orléans dans le Massif central, la Compagnie P.-L.-M. avart le privllège de se trouver en présence de puissantes entreprises de production et de transport d'énergıe, en pleın essor; aussı a-t-elle estımé que son rôle devait être de contrubuer à leur développement et à leur prospérité en s'adressant à elles pour se procurer l'énergı nécessaire à la traction électrique, plutôt que de la produire ellemême.

Le Midı et l'Orléans, conduits à s'instituer producteurs et transporteurs d'énergie, se Lrouvaient dans l'obligation de réalıser d'emblée une tranche très importante de leur programme d'électrification, afin d'assurer dès le début à leurs usines et à leurs artères à haute tensıon une utilısatıon satisfaisante. La Compagnie P -L.-M., au contrarre, décidée à ne faire appel qu'aux dısponibilités des grandes sociétès d'électricité de la région, conservalt la liberté de procéder par étapes beaucoup plus modestes, et d'altendre, pour électrifier la ligne, le moment le plus opportun.

Cetle faculté d'ajournement s'est révélée pour nous très précieuse, lorsque nous eûmes à faire face, dans ces dernières années, à des diffıcultés économıques et financières exigeant une compression énergique du budget des dépenses. De 1924 à 1926 notamment, il fallut différer impitoyablement toute dépense qui n'était pas strictement indispensable à la sécurité de l'exploitation et à l'acheminement du trafıc. Ce dernier s'étant brusquement développé avec une ampleur anormale, nécessita des dépenses considérables pour l'augmentation du débit des lignes et l'agrandissement des gares et des dépôts. Il devint dès lors impossible de trouver, dans des budgets sévèrement limités, les crédits nécessaures aux travaux d'électrification, qu'on dut par suite ralentır, et même suspendre complètement.

Sur les réseaux voisins, la situatıon était quelque peu différente. Le trafic subissait une progression bien moins rapide que sur le $P$-L.-M , et n'imposait pas un effo $t$ financier aussi consıdérable pour l'agrandissement des gares, de sorte que les budgets présentaient quelques disponibılités pour les travaux d'électrification. Ceux-cı n'auraient pu être d'ailleurs suspendus sans risquer de laisser trop longtemps improductives les dépenses fort importantes déjà engagées dans ce but.

Tels sont les motifs, d'ordre à la fors technıque et économıque, pour lesquels la traction électrique n'a pu prendre, sur notre réseau, une extensıon aussı rapıde que sur ceux du Midı et d'Orléans.

C'est en 1921 que la Compagnie P.-L.-M. décida d'électrifier la ligne de Modane, et les premières commandes pour la construction des locomotives électriques et l'installation des sous-stations furent lancées en 1922 et 1923 On avat entrepris en même lemps l'équipement des voles entre Chambéry et St-Plerre-d'Albigny, et dès le printemps de 1925, on effectuait sur cette sectıon les premiers essaıs de locomotives électrıques.

Les travaux suspendus à cette époque, en raison de la crise économique à laquelle j'a fait allusion, ne purent être repris au delà de St-Pıerre-d'Alb'gny qu'en 1927. Mais diverses difficultés et notamment les intempéries et les rigueurs de l'hiver vinrent entraver les efforts de nos entrepreneurs, et retarder les travaux, de sorte que les locomotives électriques n'ont pu parvenir jusqu'à St-Jean-de-Maurienne qu'au printemps de cette année. Elles remplacent progressivement les locomotives à vapeur sur la section de Chambéry à St-Jean-de-Maurienne, et, sauf difficultés imprévues, cette substitution sera complètement achevée dans le courant de l'été.

Au delà de St-Jean, les travaux sont poussés activement, et, si les rigueurs du prochain hiver ne se manifestent pas trop prématurément, l'équipement de la ligne jusqu'à Modane pourrait être terminé avant la fin de l'année. De toute façon, c'est seulement en 1930 que le service complet de la ligne de Chambéry à 
Modane pourra bénéficier des avantages de la traction électrique.

L'énergie nécessaire à la traction électrıque sur la ligne de Modane nous est fournie par la Société d'électro-chmme, d'électrométallurgie et des Acréries électrıques d'Ugıne, dont vous connaissez tous les belles installations et notamment l'audacieux aménagement du lac de la Grrotte

La puissance des alternateurs installés dans les usines de cette société dépasse 70000 kilowatts, et sa capacité de production atteint 250 millions de kllowatt-heures par an En regard de ces chiffres imposants, les besoins de la ligne de Modane semblent bien modestes, car la consommation annuelle ne sera guère que d'une vingtaine de millions de kilowatt-heures au début, avec des pointes de puissance de l'ordre de 10000 kilowatts.

Ce rapprochement permet de saisir avec quelle facihté notre fournisseur d'énergie couvrura nos besoins

Il fait également ressortir l'avantage qu'une entreprıse de traction électrique peut trouver, au point de vue de la sécurité de son alimentation, à n'être qu'un gros client d'un puissant producteur d'énergie, au heu d'aménager des usines pour ses propres besoins. Il est clair, en effet, qu'on n'aurait pu donner, à une usine spécialısée à la ligne de Modane, une réserve de puissance comparable à celle que présentent les nombreuses usınes génératrices de notre fournisseur, répartıes en outre dans plusieurs bassins différents.

D'autre part, tant à cause de l'importance des machınes à tenır en réserve pour farre face à des avaries accidentelles, qu'en raison des fluctuatıons consıdérables à prévoir dans la demande d'énergie sur la ligne de Modane, une usıne spécıalısée n'aurait eu fatalement qu'une utlisation peu satisfaisante En branchant, au contraire, les lignes de traction sur un important réseau alimentant des installations de nature très variée, on profite d'un facteur bien connu de toutes les sociétés de distribution d'électrıcité : c'est la diversité de la clientèle, quı vıent atténuer dans l'ensemble les irrégularités des demandes de chaque consommateur, pour assurer aux usines productrices une marche avantageuse.

Les usines de la Société d'électro-chimie sont reliées entre elles par un réseau de lignes trıphasées à 42000 volts, aboutissant au poste de Venthon, où se fait notammenr la jonctıon avec la ligne à 120.000 volts de la STEDA.

C'est également de ce poste de Venthon que partent les lignes à haute tension de la Compagnie P.-L.-M. pour lesquelles nous avons conservé la tension de 42000 volts adoptée antérieurement par notre fournisseur d'énergie.

Nos artères à 42.000 volts suivent d'abord la vallée de l'Isère, pour rejoindre la ligne de Modane près de la gare de St-Pierred'Albigny, et se diriger ensuite d'un côté vers Chambéry, et de l'autre vers Modane.

Toutes ces artères comportent sıx câbles d'aluminium constıtuant deux lignes triphasées indépendantes - normalement, les deux lignes de chaque artère fonctionnent en parallèle, mais les sections des câbles sont suffisantes pour permettre de travaller avec une ligne seulement en cas d'avarie à la seconde, sans que les chutes de tension atteignent des valeurs excessives.

Par raison d'économie, les six câbles d'une artère double sont portés par les mêmes pylônes, mais l'écartement des isolateurs est tel qu'on puisse travailler sans danger sur une ligne en maintenant l'autre sous tension

Pour la tension de 42.000 volts, on a pu se contenter d'isola- teurs à tige, sauf sur les pylônes d'ancrage el pour certaines porlées spéciales, où l'on a adopté des isolateurs suspendus.

L'énergle fournie sous forme de courants triphasés à 42.000 volts est transformée en courant contınu à 1500 volts dans huil sous-stations répartıes le long de la ligne, lous les vingt kllomètres environ, sauf sur la section de St-Jcan à Modane, où l'écartement des sous-statıons a dû ètre ramené à dix kilomètres, en rasson de la présence de rampes alteignant Jusqu'à trente millimètres par mètre.

La sous-slation de St-Pierre-d'Albigny est d'un lype spécial, parce qu'elle est chargée de régler le facteur de pussance de toute l'installation Dans ce but, la conversion du courant alternatif en courant continu s'effectue au moyen de groupes moteurs-générateurs, permetlant de régler de façon indépendante les régimes de fonctıonnement du côté alternatıf el du côté continu. Grâce aux moteurs synchrones de ces groupes, nous pouvons produire l'énergie réactıve nécessarre pour maintenır, au point de livraison de l'énergie par notre fournisseur, un facleur de puissance aussı voisin que possible de :unté, quel que soit le régime de marche de nos autres sous-stations.

Pour ces dernières, où l'on n'a plus, dès lors, à se préoccuper d'un réglage quelconque du côté alternatif, on a pu se contenter de. commutatrices, plus simples et moins coûteuses que des groupes moteurs-générateurs, et d'un rendement bien plus élevé.

Ces commutatrices produisent du courant conlinu à 750 volts, seulement, et sont groupées par deux en sérıe pour alımenter à 1.500 volts les lignes de traction La puissance nominale de chaque machine est de 1000 kllowatts, avec possibilité de fournir 1.500 kilowatts pendant deux heures, et jusqu'à 3.000 kilowatts pour des pointes de courte durée : cette faculté de surcharge est indıpensable pour permettre aux sous-stations de répondre aisément aux besoins très varıables de la traction électrıque. Ces machınes sont en outre établies pour pouvoir fonctionner en récupération sous une charge égale à leur puissance nominale, soit $1.000 \mathrm{kilo-}$ watts.

Les sous-stations sont construites pour recevoir trois groupes de deux commutatrices, solt au total 6.000 kulowatts de puissance nominale. Mais pour l'instant nous avons pu nous contenter, dans la plupart des sous-stations, de deux groupes seulement, soit 4.000 kilowatts.

Il semblerait a priorı qu'ıl eût été plus simple d'employer des commutatrices à 1.500 volts et non à 750 volts, ce qui eût évité de placer deux machines en série dans chaque groupe. Mass la construction des commutatrices de traction à 1.500 volts, alımentées en courant triphasé à 50 pérıodes, est assez délıcate, surtout pour des machines appelées à subir, comme nous l'avons dit, des sur* charges fréquentes et considérables, et devant en outre supporter impunément les violents courts-circurts qui constituent l'apa nage peu enviable, mass inéluctable, de la tractıon électrique.

On peut dire qu'actuellement, les constructeurs n'ont encore réussı à fabriquer couramment des machines de ce genre que pour des puissances nominales ne dépassant guère 750 kilowatts. En admettant même qu'on ait pu atteindre 1.000 kilowatts, il eût fallu installer, dans nos sous-stations, le même nombre de commutatrices dans les deux hypothèses, qu'elles fussent à 1.500 volts ou à 750 volts. Mais le nombre des tableaux et des jeux d'appareillage eût été doublé, car ıl suffıt d'un seul tableau el d'un seul appa* reillage pour un groupe de deux commutatrices à 750 volls en série.

En résumé, la solution que nous avons adoptée était donc, dans l'espèce, la plus simple et de beaucoup la moins coûteuse. Mais il faudrait bien se garder de généraliser cette conclusion. 
Le problème se fûl posé, en effet, de façon toute différente, si nous avions dû être alimentés, comme dans la région du Sud-Est, par exemple, en courants trıphasés à vingt-cınq pérıodes au lieu de cinquante, ce qui eût rendu possible la construction de commutatrices à 1.500 volts de grande puissance. Il est permıs d'espérer d'autre part que, même pour la fréquence de 50, les progrès de la technique permettront plus tard de dépasser largement la limite de puissance dont il faul se contenter actuellement pour les commutalrices à 1.500 volts.

Il est à crandre toutefois que ces machines n'aient dans l'avenir que des débouchés assez restreints, en raison du développemenl de la fabrication des redresseurs à vapeur de mercure, dont les mulliples avantages vous sont bien connus. Leur seul inconvénient est de ne pas se préler à la récupération, mais il est largement compensé, dans la plupart des cas, par la supériorité de leur rendement, surloul à farble charge . ce n'est donc que dans des conditions assez spéciales, par exemple sur les lignes à très longues déclivités, que le bilan final pourra être favorable aux commutatrices.

Toutes nos sous-stations constituent, pour les lignes doubles à haute tension, des postes de sectionnement et de couplage en parallèle, sur un double jeu de barres omnibus.

Pour écouler à la terre les charges statiques quı peuvent prendre naissance sur les lignes à haute tensıon par suite de phénomènes atmosphériques, on a branché, sur chaque barre ommbus à 42.000 volts, une bobine de réactance à noyau de fer, immergée dans l'huile, et dont l'extrémité est à la terre. Grâce à leur faıble résistance, ces bobınes peuvent laisser passer un courant contınu suffisamment important pour écouler rapidement à la terre une charge statique accidentelle, tandıs que leur forte réactance rend absolument insignifiantes les futes en courant alternatif à 50 périodes.

Quant à la protection contre les surtensions présentant le caractère d'ondes à front ra1de ou à haute fréquence, et provenant soil de décharges atmosphériques, sort de mise accidentelle d'un câble à la terre, soit même simplement de l'ouverture brusque d'un disjoncteur sous charge, elle est assurée, dans la plupart des sous-stations, par des amortisseurs d'ondes · cet apparell est constitué par un condensateur branché entre deux bobınes de self insérées sur la barre omnibus, la bobıne placée du côté des lignes étant elle-même shuntée par une résistance. Toutefors, dans les sous-statıons de St-Plerre-d'Albigny et de la Praz, ce dispositif est remplacé par des parafoudres à oxyde de plomb, installés sur les entrées et les départs des lignes à haute tension. Toutes ces lignes sont en outre munies de câbles de terre placés au sommet des pylònes.

Au point de vue des surmlensités, la protection est réalısée, par un système très complet de relais divers, soit à action directe, soit à actıon différentıelle, soit à retour d'énergie provoquant le déclenchement séleclif des disjoncteurs à 42000 volts, de façon à isoler un tronçon de ligne défectueux sans interrompre l'alimentation des sous-stations par la l gne restée saine.

Du côté des circuits à courant continu, la protection contre les courts-circuits est assurée par des disjoncteurs extra-rapides, installés tant sur les départs à 1.500 volts que sur le pôle négatif de chaque groupe de commutatrices. A l'inverse des disjoncteurs de feeders, qui coupent drectement le curcurt, ceux des groupes de commutatrices se bornent à insérer dans le circuit une résistance pour limıter immédıatement le débit; la coupure est ensuite effectuée par un disjoncleur ordinaire, placé du côté positif. On réalise ainsi la coupure en deux temps, avec de moindres risques pour l'appareillage, et sans qu'il se produise de coup de feu aux collecteurs des commutatrices, d'aulleurs protégés par des écrans spéciaux.

En fait, cette protection s'est toujours montrée entièrement efficace, même en cas de court-circuit franc, provoqué intentıonnellement au droit de la sous-station, pour éprouver la valeur des apparells dans les conditions les plus dures.

Je me souviens notamment d'avoir fait exécuter cette expérıence en présence de l'éminent spécialıste de l'électrifıcatıon des Chemins de fer suisses, mon ami le docteur Hubor, quı manifestait son étonnement de me voir infliger un pareil traitement à des commutatrices dans le seul but de lui être agréable : ll fut quelque peu déçu de constater que le court-circuit ne fut décelé que par le déclenchement du dısjoncteur, alors qu'll avaıt eu l'espoır d'assister à un feu d'artıfıce impressionnant.

Avec le courant continu à 1.500 volts, on peut employer indifféremment la prise de courant sur ligne aérienne ou sur rall conducteur, dit " troisième rall".

Au point de vue économique, la ligne aérienne est plus avantageuse pour l'électrıfıcatıon des lıgnes à trafic relatıvement peu important, pour lesquelles on peut se contenter de caténaires simples et légères. Mais sur les lignes à fort trafic, où il faut pouvoir capter à des vitesses très élevées des courants de très forte intensité, on est conduit à recourır à des caténaures plus compliquées et à les compléter par d'importants feeders pour obtenır la conductiblité totale requise. les dépenses sont alors du même ordre que pour l'installation d'un rall conducteur.

Ce dernier présente d'ailleurs de précieux avantages, car la pose et l'entretien en sont beaucoup plus faciles que dans le cas des lignes aérıennes, exigeant l'emploi d'échelles et d'échafaudages roulants. Les agents de la vole peuvent visiter et entretenir le rall conducteur au cours de leurs tournées de surveillance, comme ils le font pour les ralls de roulement eux-mêmes. En cas d'accident, les avaries sont bien moins graves qu'avec les lignes aériennes, et les réparations nécessaires peuvent être exécutées bien plus rapidement. Toutes ces qualités sont partıculıèrement intéressantes pour l'électrıfıcatıon des lignes à fort trafıc, où le passage de nombreux trains gêne les opératıons d'entretıen, et exıge une remise en état aussi rapide que possible de tout organe avarıé Telles sont les considérations primordıales qui ont décidé la Compagnie P.-L - M à faire l'essai du troısième raul sur la ligne de Modane, de préférence aux caténaires aériennes adoptées par le Midi et l'Orléans.

Mais ce type de conducteur présente, par contre, certains inconvénients que ne possèdent pas les lignes aérıennes Tout d'abord, il risque de constituer un obstacle dangereux pour la circulation du public dans les gares, et du personnel sur les voies. On peut y remédier efficacement en le protégeant sur toute sa longueur par une gaine en planches, quı ménage simplement une rainure pour le passage des frotteurs des locomotives : avec une protection de ce genre, convenablement étudiée, le risque de contact accidentel est pratıquement supprimé. En pleine voie, les rails conducteurs sont d'alleurs installés systématiquement dans l'entre-voie, afin de facilter la circulation des agents sur les côtés de la ligne. Mais cette disposition ne peut être réalisée unformément dans les gares, où la présence d'aiguilles et de croisements oblige à installer certains tronçons du rail conducteur à l'extérieur des voles.

Au droit de ces appareils (aiguilles et croisements), le troisième rall doit forcément être interrompu sur une certaine longueur : les deux tronçons de conducteur situés de part et d'autre de la coupure sont alors reliés par un câble armé souterrain, pour rétablir la continuité du circuit à 1.500 volts. 
Tant que la longueur de cette coupure reste inférieure à l'écartement des frotteurs placés aux deux extrémités de la locomotive, le passage s'effectue sans que la prise de courant subisse d'intreruption, et tout se passe comme si le troisième rall ne présentail aucune solution de contunuté. Il en est ansi, notamment, dans le cas d'aigulles isolées, ou suffisamment éloignées les unes des autres.

Mais lorsque les appareils de voie se succèdent à de farbles intervalles, les coupures deviennent alors trop longues pour qu'on puisse éviter de priver momentanément les locomotives de courant. Dans les gares peu importantes, l'inconvénient n'est généralement pas grave, parce que ces coupures anormales sont peu nombreuses, et que leur longueur est assez rédurte pour que les machınes puissent aisément les franchir au lancé. Au surplus, si elles tombent accidentellement en panne au milieu d'une lacune par suite d'une fausse manœuvre, on peut les dépanner très rapidement au moyen d'un câble muni de poignées isolantes, avec lequel on établit une connexion temporare entre les frotteurs et le tronçon de rall conducteur le plus voisin. Au besoin, on peut rédurre ce risque de panne en remaniant les apparells de vole afın d'éviter les coupures de longueur exagérée, la disposition assez simple des voles de service des petites gares se prète facılement à des modifications de ce genre.

Mais cette solution devient inapplicable dans les grandes gares, ou l'enchevêtrement des appareils de voie rendrait un tel remaniement extrêmement onéreux, et parfors même impossible, et où le risque de panne deviendrait inadmissible en raison du nombre et de la grande longueur des coupures. On est alors obligé d en revenir à la lıgne aérıenne, comme nous avons dû le farre en particulier en gare de Chambéry, où le troisıème rall n'a été installé nulle part, même sur les vores principales.

Dans d'autres gares importantes, comme Montmélian et SantJean-de-Maurienne, on a pu conserver le troisième ral sur les vores principales, tout en installant des hgnes aériennes sur les voles de service, afın d'éviter des remaniements trop onéreux. A Modane, par contre, on ne pouvart envisager parelle solution, à cause de la présence des lignes aériennes triphasées pour l'alımentation des locomotives italiennes : on a donc cté forcé de prévoir les remanements de voles nécessaires pour permettre l'implantation du troisième rall sur les voies de service.

Cette dualité du mode de prise de courant, normalement sur troisième rail, et accessoirement, sur fil aérien, a entraîné l'ınstallation de zones de transition, dans lesquelles coexistent les deux types de conducteurs, afin de permettre aux locomotives de passer de l'un à l'autre sans interrompre ni même ralentır leur mouvement. De multiples expériences, exécutées dans des conditions très variées, nous ont montré que cette transition pouvart s'effectuer sans aucune difficulté, et à des vitesses bien supérıeures à celles quı seront pratıquement atteintes à la traversée de ces zones.

Les dispositions adoptées permettent donc de remédier efficacement à l'inconvénient des coupures de grande longueur dans le troisı̀me rail

A l'époque où il fut question d'entreprendre des essals préliminaires de ce type de conducteur sur la section de Chambéry à St-Pierre-d'Albigny, on admettait que les coupures de grande longueur, franchissables seulement au lancé, devarent être systématiquement écartées Cette opınion était fondée sur le résultat d'expériences effectuées en Amérique par la General Electrıc Company, sur la demande des ingénieurs de la Compagnie d'Orléans. Ces expériences paraissaıent avoir établı que, si l'on ne prenaıt pas la précaution de couper le courant sur la locomotive avant d'abor- der une telle lacune, il se produranl, au moment où le frotlew arrière de la locomolive abandonnaıl le trossième rail un ar! de ruplure important, susceptible d'attemdre les boîtes onl: châssıs de la machıne, en provoquanl un court-circuit asse? violent pour farre déclencher les sous-stations. Aussi avions-noy exécuté sur la sectıon de Chambéry à St-Pierre-d'Albıgny lou les remaniements d'apparels de vore ct toutes les installation de tronçons de lıgne aérienne nécessaures pour faire disparailti complètement les coupures de grande longueur dans le trossièmi rail.

Mais dès que cette section fut équipée, nous entreprîmes auss: tôt de rééditer les expériences américaines, dans les conditıons le plus varıées, tant au pointdevue de la-vilesse que de l'intensité des: courants captés, qui devarent être coupés par le frolleur lu1-mêmen au moment où ll quittail le trosième rall. A notre grand éton nement, le phénomène annoncé ne s'est jamais produit : l'arm de rupture n'a rien d'inquiélant, et s'étemt de lui-même sinn sauter à la masse On peut donc en conclure qu'avec les dispoś trons adoptées par notre trossième rail el nos frotteurs de loce motives, il est inutule de couper le courant sur la machine avan: de franchir au lancé une lacune de grande longueur dans b troisième rall.

Cette conclusion est partıculı̀rement intéressanle. Car ell: nous a permis, lors de la reprise des travaux d'électrification au de là de St-Pierre-d'Albigny, d'accepter de telles lacunes dans 1 rall conducteur, toutes les fors qu'll s'agissait de coupures suscep tibles d'être arsément franchies au lancé, sans risque de panne, par des machines effectuant des manœuvres. Nous avons pu évite ansi bien des remaniements d'appareıls, et certains tronçons di lignes aériennes, dont l'exécution eûl entrainé au total des dépen ses fort élevées.

Le trossième rall installé sur la ligne de Modane est du tỵp ordınaıre, "à conlact par dessus ", tel qu'on le rencontre par exem ple sur le Chemın de fer Métropolitain de Paris, ou sur notr ligne de Chamonix, c'est-à-dıre que les frotteurs viennent s'applt quer sur la face supérieure du rail.

On a souvent prétendu qu'avec cette disposition la prise d courant devenat fatalement très défectueuse en cas de nerge, surtout de verglas, et que cet inconvénient ne pouvait être évilt qu'en adoptant un type de ral conducteur différent, avec contad sur le côté ou mème par dessous. En réalité, les brillants felir d'artıfice que l'on peut observer par temps de neige ou de vergh: sur certaines lignes électrifiées avec un rail à contact supéreul proviennent uniquement du fait que les frotteurs sont d'un type défectueux. ils caressent mollement le rall conducteur au leet de s'appuyer énergıquement sur luı L'expérıence déjà longue de no tre ligne de Chamonix montre bien qu'on peul assément caplet des courants intenses sur un trosstème rall à contact supériellr malgré les intempérıes, à la condıtıon d'exercer une pression suffisante sur les frotleurs au moyen d'arr comprimé, el on les munissant au besoin de lames d'acier qui débarrassentle rall de toute couche de verglas. Avec de parenls dispositiss nous n'avons jamass éprouvé aucune difficulté sur la ligne de Chamonix, où les intempérıes sont cependant particulièrement fréquentes et sévères Leur application est d'allleurs bien plus facile avec le type ordinaire de trossième ral, de sorte que les variantes à contacl latéral ou à contact par dessous ne semblınt présenter, en défintive, aucun intérêt bren marqué.

Le rasl à contact supérieur, utllisé couramment pour des tensions de 600 à 800 volts, peut être adapté sans difficulté à la tension de 1.500 volts : il suffit de le placer sur des isolateurs convenables. 
Ceux de la ligne de Modane sont conslitués par de gros blocs en grès, en porcelaine, ou en basalte fondu.

Ces deux dermers types domnent un isolement melleur, mars on peut atténuer largement l'infériorté que présentent à cet égard les isolateurs en grès en les trempant simplement dans un bann de paraffine : ce tratement est peu coûteux, et l'expérience monlte que l'amélioration obtenue se mantient malgré les intempétios

Au demourant, les qualités isolantes de ces blocs ne sont pas seules 'n jou leur résistance aux chocs n'a pas moins d'mportance car les tiaverses sur lesquelles ils reposent leur transmettent de violentes ríaclions lors du passage des trans lls sont en outre soumis à des épreuves encore plus brutales daus les manutentions qui précédent leur mise cu place

Or les isolateurs en gi ès paraissent supporter ces trailements sévères beaucoup plus facilcment que ceux en basalte fondu ou en porcelainc. Ils sont d'alleurs d'un prix sensiblement moins élevé, mème avec les frais de traitement à la paraffine L'écarl augmente encore si l'on considère non pas les prix en usine, mass les prix de levient des isolateurs mis en place, auquel cas 1 faut temr comple du nombre des isolateurs brisés au cours des manulentions. Il scmble donc que, pour l'mstant du moins, on serall condunt à donner la préférence aux isolateurs en grìs paraffiné, loul en espérant que l'on pourra farre encore mieux dans lavenur

L'adoption du troisième rail à 1.500 volts sur la ligne de Modane constutuant lune des particularilés les plus originales de cette électrification, j'ai cru devorr, pour celle raison, entrer dans quelques développements à ce sujet; mais je crains maintenant d'avorr dépassé la mesure. Aussi m'abstıendraı-je de vous entretenır d'autres détals de cel équipement comme le montage de la gaine de protection du troisième rail sur cales isolanles encastrées dans son profil, ou le dispositif de sectionnement des conducteurs à 1.500 volts, ou l'éclissage électrique du trolsième rail el des rails de roulemenl. Sans doute aurais-je pu vous signaler à cette occasion plusieurs dispositions nouvelles, susceptibles d'intéresser au mons certans spécialıstes, mais je risquerais d'abuser ansi de votre bienveillance 11 me paraît donc préférable d'aborder de suite la dernière partıe de mon exposé, en vous parlant des locomotıves électriques.

Les locomotives électriques de la ligne de Modane appartiennent à deux classes distınctes ' d'une part, les machines rapıdes, et d'autre part celles affectées aux trams à vitesse modérée (trains omnibus de voyageurs, trains de messageries et trains de marchandises).

L'adoption de machines capables d'assurer indifféremment les trains de ces trois dernières catégories présentait pour nous un grand intérêt. En rasson du prix élevé des locomotives électr1ques, il était, en effet, indispensable de chercher à réduire au minimum l'effeclif nécessarre : on ne pouvart y parvenir qu'en employant le même type de machines à des services assez variés, pour augmenter le plus possible leur utilısation journalı̀re.

Le problème de la construction d'un seul type de locomotive répondant à la fols aux besoins de lous les services, sauf celui des rapides, se présentail toutefors dans des conditions particulièrcment difficiles sur la ligne de Modañe, en raison de son profil très variable. Jusqu'à Aiguebelle, c'esl presque une ligne de plaine; puis apparaissent des rampes de plus en plus forles à mesure qu'on s'élève dans la haute vallée de l'Arc. Les déclivités ne sont d'abord que de 10 à 15 pour mille jusqu'à St-Jean-deMaurienne, mais elles attergnent ensuite 20 à 25 pour mille avant
St-Michel, et enfın 30 pour mille au delà. En sens inverse, la présence de pentes aussi fortes sur une pareille longueur ımposaıt nalurellement le freinage électrıque par récupératıon, ce qui compliquart encore le problème.

En raison de cette complexilé du programme à remplir, nous nous sommes adressés, pour l'étude et la fabrication de ces machines, à trois groupements de constructeurs, chaque groupement ayant à lıvrer une sérıe de dix machines, dont la première serait essayée et mise au point avant de poursuivre la fabrication des suvantes.

Nous avions spécifié à ces constructeurs qu'une seule machıne aurait à remorquer un train de marchandıses de 800 tonnes de Chambéry jusqu'à St-Jean, et que la même charge devrart ĉtre enlevée au delà, sur les rampes de 20 à 30 pour mille, avec le renfort d'une deuxième locomotive du même type Les machınes quı nous ont été livrées paraissent devoir remplır largement ce programme, tout en assurant par alleurs dans d'excellentes conditions le service des trains de voyageurs omnibus et des trains de messageries.

Les locomotives de ces trois séries comportent uniformément six essieux moteurs encadrés entre deux essieux porteurs, mais avec des dispositions assez différentes d'un constructeur à l'autre. Les machines fournies par les Constructions Electriques de France et la Société Alsacienne n'onl qu'une seule caisse, reposant sur deux trucks, chacun d'eux étant monté sur trols essieux moteurs et un bissel Celles des autres constructeurs sont au contraire constituées par deux unités indépendantes, avec deux carsses distınctes, les châssis des deux unités étant reliés par un robuste attelage à rotule sphérıque. Parmı ces dernières locomotives, celles de la Compagnie Electro-Mécanique et de la Compagnie de Fives-Lille possèdent sous chaque caisse trois essieux moteurs et un bissel directeur; sur celles de la Compagnie Thomson-Houston el des Etablissements Schneider, les deux essieux moteurs arrière de chaque unité sont seuls montés sous le châssis principal portant la caisse, tandis que le troisième essieu moteur forme avec l'essieu porteur un truck articulé.

La puissance de ces machınes varie de 2.300 à 2.400 chevaux

Leurs moteurs sont tous du type usuel, dit type tramway avec suspension "par le nez".

Pour les machines de rapides nous avons, au contraire, jugé indispensable d'expérimenter d'autres types de transmission, avant de prendre parti; nous avons donc demandé à plusieurs constructeurs de nous livrer chacun une seule locomotive d'essai, afın de procéder à des comparaisons. Ces expérıences, entreprises en 1925 sur la section de Chambéry à St-Pierre-d'Albigny, ont conduit à donner la préférence aux machines à moteurs jumelés, avec transmission par arbre creux entourant l'essieu moteur.

Deux locomotives de ce type ont pris part aux essais, l'une entièrement fabrıquée par la Socièté Alsacienne, tandis que la Socièté Oerlikon avait fourni les moteurs et l'équipement électrique de la seconde, dont la partie mécanique étart construite par la Compagnie des Batignolles et la Compagnie Générale de Construction de Locomotives à Nantes.

Les dispositions d'ensemble de ces deux machines sont identıques, avec caisse unique reposant sur deux trucks, chacun d'eux comportant deux essieux moteurs et un bogie drrecteur. Ces locomotives ne diffèrent entre elles que par l'appareillage électrique, el par les systèmes de transmission entre les arbres creux et les roues motrices. 
Leur étude avait été faite en vue de remorquer des trans de 500 tonnes à des vitesses légèrement supérıeures à celles des rapıdes actuels, ce quı avart condùt à leur donner une pussance d'environ 2600 chevaux

Encouragé par les excellents résultats obtenus aux essais j'ai pensé que ce programme étalt trop modeste, et qu'on pouvait envisager sans difficulté des machınes beaucoup plus puissantes, susceptibles de permettre une majoration considérable des charges et des vitesses actuelles.

$J$ 'ai donc demandé au groupement Batıgnolles-Nantes-Oerlikon d'étudier une nouvelle locomotıve construite suivant les mêmes principes que la machine d'essai, mass comportant six essieux moteurs au heu de quatre. En conservant les moteurs de la machine primitive, on pouvalt aunsı majorer d'emblée de 50 pour cent la pussance de la locomotive.

Mais il m'a paru possible de farre encore mieux : j'ai invité en conséquence la Société Oerlıkon à reprendre l'étude du moteur luı-même, de façon à en augmenter la puissance, et à l'adapter aux très grandes vitesses. Cette tentatıve a pleınement réussi, car la puissance des nouvelles machınes a pu ainsi être portée à 5400 chevaux, sort le double de la puissance de la locomotive d'essai, bien que le nombre des moteurs n'ait été majoré que de moitié.

La première des quatre locomotives commandées sur ce nouveau type vient de nous ètre livrée tout récemment, de sorte que nous n'avons pas encore eu le temps de procéder à des essals complets. Il serait par surte prématuré de formuler dès maintenant une opinıon précıse sur les résultats qu'on peut attendre de la mise en service de ces nouvelles machines. Il est cependant permis de dire que les premières impressions sont excellentes, et que nos espérances ne paraissant pas devoir être déçues bien au contraire.

D'après les essais des moteurs à l'usine, ces locomotives devraient pouvoir remorquer un rapide de 700 tonnes à la vitesse de 90 kilomètres à l'heure en rampe de 8 milımètres par mètre, et de 110 kilomètres à l'heure en palier. Si les expérıences auxquelles nous allons procéder sur la ligne confırment ces résultats, de tels engins permettraient par exemple de gagner environ une heure entre Marselle et Nice, el plus d'une heure ent Parıs et Dijon, par rapport aux horaures les plus accélérés d du service actuel, tout en portanl la charge des rapides de 50 à 700 tonnes. Sur la ligne de Modane, en raison des sujétio service, et à cause du fauble développement des portions lignes franchissables à grande allure, on ne peul nalurellemer pas escompter des résultats aussi sensationnels : quoi qu'il soit, ces machines pourront remorquer des rapides beaucou plus lourds qu'actuellement, en réalısant partout la vitesse plus élevée que puisse autoriser la constitution de la voie.

J'ai quelque peu surpris mes bons amis d'Amérıque en le annonçant l'an dernier que la Compagnie P.-L.-M. allart détenir record de puissance pour les locomotives électriques. Ils m'o déclaré que je devals faire erreur, et que ce record reste certainement leur apanage, notamment avec les grosses machin de 7.000 chevaux, qui remorquent sur le Virginian Railw des trains de charbon de plus de 8.000 tonnes. Je leur ar répon que je connalssals bien ces machınes pour les avoir vues construction à Pittsburgh, mais qu'elles se composatent réalité de trous unités indépendantes, dont chacune ne développ même pas la moitié de la puissance de la nouvelle locomoty du P.-L -M.

Il est clair, en effet, qu'en atlelant l'une derrière l'autre nombre quelconque de machines fonctıonnant en unités multip comme les automotrices d'un train de banlieue, on pourr attemdre au total telle pussance qu'on voudrait, et ba aisément tous les records : mals 1 l s'agit là d'un véritable train locomotives, et non d'une machine isolée.

Il existe déjà, tant en Europe qu'en Amérique, d'autres $]$ motıves électriques à caisse unique développant une puissa bıen supérıeure à celle d'une unıté ısolée du Virginıan Railw Mars la puissance indıviduelle d'aucune de ces machines n'atte encore celle de la nouvelle locomotive du P.-L.-M. auquel revi bien pour l'mstant, n'en déplaise aux Américains, la glo toujours éphémère d'un record mondial.

\section{(Conférence faute à la Chambre de Commerce de Grenoble 5 juillet 1929.)}

